Nor mal i zed St ress I nt ensi ty Fact or Range Sol ut i ons of an I nner-Surface Ci rcumf er ent i al Crack i n Thi n- to Thi ck- Wall ed Cyl i nder under Ther mal Stri pi ng by Sem-Anal yt i cal Numer i cal Met hod

\begin{tabular}{|c|c|}
\hline 著者 & MESH I Toshi yuki, WATANABE Kat suhi ko \\
\hline $\begin{array}{l}\text { jour nal or } \\
\text { publ i cat } i \text { on } \mathrm{title}\end{array}$ & Jour nal of Ther nal Stresses \\
\hline vol une & 27 \\
\hline nunber & 3 \\
\hline page $r$ ange & $253-267$ \\
\hline year & 200403 \\
\hline URL & ht t p: //hdl . handl e. net /10098/1129 \\
\hline
\end{tabular}


T. Meshii, et al., Journal of Thermal Stresses, Vol. 27, No. 3, pp. 253 - 267 (2004. 3).

\title{
Normalized Stress Intensity Factor Range Solutions of an Inner-Surface Circumferential Crack in Thin- to Thick-Walled Cylinder under Thermal Striping by Semi-Analytical Numerical Method
}

\author{
Toshiyuki MESHII $^{\mathrm{a} *}$ and Katsuhiko WATANABE ${ }^{\mathrm{b}}$ \\ ${ }^{\mathrm{a}}$ Department of Mechanical Engineering, Fukui University, 3-9-1 Bunkyo, Fukui, Fukui, 910-8507, Japan \\ ${ }^{\mathrm{b}} 1^{\text {st }}$ Division, Institute of Industrial Science, University of Tokyo, 4-6-1 Komaba, Meguro-ku, Tokyo, 153-8505, Japan \\ *Correspondent, E-mail: meshii@mech.fukui-u.ac.jp, FAX : +81-776-27-8468
}

\begin{abstract}
The normalized stress intensity factor (SIF) range of an inner-surface circumferential crack in a thin- to thick-walled finite-length cylinder under thermal striping was considered in this paper. The edges of the cylinder were rotation-restrained and the outer surface was adiabatically insulated. Inner surface of the cylinder was heated by a fluid with sinusoidal temperature fluctuation. An analytical temperature solution for the problem and our semianalytical numerical SIF evaluation method for the crack were combined, and as a result, it was shown that the transient SIF solution can be expressed in a generalized form by dimensionless parameters such as mean radius to wall thickness ratio, Biot number, normalized striping frequency and Fourier number. Finally, normalized SIF ranges for the 1st cycle and steady state were given for these dimensionless parameters in tables for mean radius to wall thickness ratio of 10,5 and 1.
\end{abstract}

Key Words: Fracture Mechanics; Stress Intensity Factor; Thermal Stress; Thermal Striping; Circumferential crack

\section{INTRODUCTION}

Temperature fluctuation which occurs in a region where hot and cold fluids incompletely mix sometimes causes damage in structures. For example, a coolant leakage in a nuclear power plant due to thermal fatigue induced by sinusoidal coolant temperature fluctuation (thermal striping) was reported recently [1]. Since thermal striping is 
T. Meshii, et al., Journal of Thermal Stresses, Vol. 27, No. 3, pp. 253 - 267 (2004. 3).

usually uncontrollable and since the damage accumulates quickly due to rapid sequences of cycles, we thought it important to collect stress intensity factor (SIF) solutions under thermal striping for various crack configurations and prepare for accidents in the future. Therefore in this paper, we derive the SIF solution for an inner-surface circumferential crack in a finite length cylinder (hereafter, simply called as circumferential crack) under thermal striping (Figure 1) as the first step. The cylinder of initially uniform temperature $T_{\mathrm{m}}(t<0)$ is insulated adiabatically outside and is heated inside by a fluid of $T_{\mathrm{m}}+T_{\mathrm{f}}(t)=T_{\mathrm{m}}+\Delta T \sin \omega t(t \geq 0)$ with constant heat transfer coefficient $h$. The edges of the cylinder are rotation-restrained and the crack is located at the midpoint of the cylinder length. This SIF for the problem is affected by various factors such as cylinder configuration, heat transfer conditions and thermal striping frequency, etc. Thus we aim to present the desired SIF solution in a normalized form to minimize the number of non-dimensional parameters.

In the past, transient SIF under thermal shock (a case in which fluid temperature is given as a step function; i. e. following the description in Figure 1, $T_{\mathrm{f}}(t)=-2 \Delta T$ for $t \geq 0, T_{\mathrm{f}}(t)=0$ for $t<0$ ) have been obtained analytically for a crack in coated plates and shells [2], [3], and for a circumferential crack in an infinite-length cylinder [4], [5]. Besides these pioneering works, recently we proposed a semi-analytical numerical method to systematically evaluate the effects of various parameters (including cylinder length) affecting the SIF for the circumferential crack in Figure 1, for cases under arbitrary radial temperature distribution of $T_{\mathrm{m}}+u(r, t)$, and when we applied the method to the thermal shock problem, the results showed that the transient SIF for a circumferential crack in a finite length cylinder can be larger than that under long cylinder assumption [5]. Taking also this fact into account, we apply our semi-analytical numerical method for our purpose. Our method was derived by replacing the problem with isothermal problems and is suitable for studying the effects of the various structural and heat transfer parameters on the desired SIF. The results obtained from the parametric studies were that (i) the SIF is proportional to Young's modulus $E$, coefficient of thermal expansion $\alpha$, square root of cylinder wall thickness $W^{1 / 2}$ and fluid temperature change $2 \Delta T$, (ii) the structural parameters that affect the SIF are the cylinder mean radius to wall thickness ratio $r_{\mathrm{m}} / W$, aspect ratio $H / W$ and, though small, Poisson's ratio $v[6]$

By our method, the SIF at a specific time and the SIF range in a specific period can be evaluated once the 
cylinder temperature change from the initial state $u(r, t)$ is known. Therefore we thought if we could obtain $u(r, t)$ under thermal striping and express it in a normalized form, we would be able to normalize the desired SIF and SIF range under thermal striping. Since we are concerned with the damage accumulated due to rapid sequence of cycles, we focus on the SIF ranges under thermal striping, considering the Paris law [7]. In the following, we first introduce our $u(r, t)$ solution under thermal striping [8] and then normalize it by non-dimensional parameters. Subsequently, we normalize the transient SIF and present the SIF ranges at the 1st cycle and for steady state for various dimensionless parameters in tables for the cases of $r_{\mathrm{m}} / W=10,5$ and 1.

\section{NORMALIZATION OF ANALYTICAL TRANSIENT TEMPERATURE SOLUTION UNDER THERMAL STRIPING}

\section{Analytical Transient Temperature Solution under Thermal Striping}

Heat conduction problem of a hollow and long cylinder whose inner and outer radii are $r_{\mathrm{i}}$ and $r_{\mathrm{o}}$, respectively, was considered (Fig. 1). The cylinder temperature $T_{\mathrm{m}}$ is uniform at time $t<0$ and the temperature change from the initial state is defined as $u(r, t)$. The cylinder is adiabatically insulated on the outer surface and the inner surface is axisymmetrically heated by a fluid whose temperature is $T_{\mathrm{m}}+T_{\mathrm{f}}(t)$ with a constant heat transfer coefficient $h$. The material constants of the cylinder, such as thermal conductivity $\Lambda$ and thermal diffusivity $\kappa$ are assumed to be temperature independent.

The partial differential equation (PDE) to be solved, boundary conditions (BCs, second type) and initial condition (IC) are as follows [9]:

$$
\begin{array}{cc}
\frac{\partial u}{\partial t} & =\kappa\left(\frac{\partial^{2} u}{\partial r^{2}}+\frac{1}{r} \frac{\partial u}{\partial r}\right) \\
\left.\Lambda \frac{\partial u}{\partial r}\right|_{r=r_{o}}=0 & 0<t<\infty \\
-\left.\Lambda \frac{\partial u}{\partial r}\right|_{r=r_{\mathrm{i}}}=h\left(T_{\mathrm{f}}-u\right) & 0<t<\infty
\end{array}
$$


T. Meshii, et al., Journal of Thermal Stresses, Vol. 27, No. 3, pp. 253 - 267 (2004. 3).

$$
u(r, 0)=0 \quad r_{\mathrm{i}} \leq r \leq r_{\mathrm{o}}
$$

Since we could not find the analytical solution when $T_{\mathrm{f}}(t)=\Delta T \sin \omega t(t \geq 0)$, we obtained the concrete $u(r, t)$ for this case [8]. We introduced functions $U(r, t) \equiv u(r, t)-T_{\mathrm{f}}(t)$ and $f(t) \equiv-d T_{\mathrm{f}}(t) / d t$, and constants $U_{0} \equiv-T_{\mathrm{f}}(0)$ and $\alpha_{0} \equiv h / \Lambda$ to convert the nonhomogeneous BC (Eq. (3)) to the homogeneous type [10]. As a result, the PDE itself becomes nonhomogeneous. Thus, we applied the eigenfunction expansion method [10] to solve the PDE and obtained the general solution for $T_{\mathrm{f}}(t)$ as follows [8]:

$$
u(r, t)=T_{\mathrm{f}}(t)+\sum_{n=1}^{\infty} R_{n}(r)\left\{U_{0} e^{-\kappa \rho_{n}^{2} t}+\int_{0}^{t} e^{-\kappa \rho_{n}^{2}(t-\tau)} f(\tau) d \tau\right\}
$$

The eigenvalues $\rho_{n}$ are positive roots of the following equation $\left(\rho_{n} \leq \rho_{n+1}\right)$.

$$
J_{1}\left(\rho_{n} r_{\mathrm{o}}\right)\left[Y_{0}\left(\rho_{n} r_{\mathrm{i}}\right) \alpha_{0}+Y_{1}\left(\rho_{n} r_{\mathrm{i}}\right) \rho_{n}\right]=Y_{1}\left(\rho_{n} r_{\mathrm{o}}\right)\left[J_{0}\left(\rho_{n} r_{\mathrm{i}}\right) \alpha_{0}+J_{1}\left(\rho_{n} r_{\mathrm{i}}\right) \rho_{n}\right]
$$

By applying the orthonormality of the cylinder functions [11] and by introducing $\beta_{n} \equiv J_{1}\left(\rho_{n} r_{\mathrm{o}}\right) / Y_{1}\left(\rho_{n} r_{\mathrm{o}}\right)$, the eigenfunction $R_{n}(r)$ was derived as follows:

$$
R_{n}(r)=\frac{2 \alpha_{0} r_{\mathrm{i}}}{\rho_{n}^{2}} \times \frac{\left\{J_{0}\left(\rho_{n} r_{\mathrm{i}}\right)-\beta_{n} Y_{0}\left(\rho_{n} r_{\mathrm{i}}\right)\right\}\left\{J_{0}\left(\rho_{n} r\right)-\beta_{n} Y_{0}\left(\rho_{n} r\right)\right\}}{r_{\mathrm{o}}{ }^{2}\left\{J_{0}\left(\rho_{n} r_{\mathrm{o}}\right)-\beta_{n} Y_{0}\left(\rho_{n} r_{\mathrm{o}}\right)\right\}^{2}-r_{\mathrm{i}}^{2}\left\{1+\left(\alpha_{0} / \rho_{n}\right)^{2}\right\}\left\{J_{0}\left(\rho_{n} r_{\mathrm{i}}\right)-\beta_{n} Y_{0}\left(\rho_{n} r_{\mathrm{i}}\right)\right\}^{2}}
$$

where $J$ and $Y$ represent the Bessel functions of first and second kinds, respectively. Suffixes 0 and 1 for $J$ and $Y$ represent the order of these functions.

Thus the function shape of $u(r, t)$ for the cases of $T_{\mathrm{f}}(t)=\Delta T \sin \omega t(t>0)$ was obtained as follows:

$$
\frac{u(r, t)}{\Delta T}=\sin \omega t+\sum_{n=1}^{\infty} \frac{\omega R_{n}(r)}{\left(\kappa \rho_{n}^{2}\right)^{2}+\omega^{2}}\left\{\kappa \rho_{n}^{2}\left(e^{-\kappa \rho_{n}^{2} t}-\cos \omega t\right)-\omega \sin \omega t\right\}
$$

Note that the obtained $u(r, t)$ expressed as Eq. (8), which was to satisfy the PDE for $t>0$ and $r_{\mathrm{i}}<r<r_{\mathrm{o}}$, directly gives the value on the boundaries which satisfy the BCs of second type. This nature of the solution is preferable for applying the solution in calculating the concrete values near the boundaries.

\section{Normalization of Temperature Distribution Solution}

First we normalize the $n$th eigenvalue as $\rho_{n} r_{\mathrm{i}} \equiv x_{n}$ and the heat transfer parameter as $\alpha_{0} r_{\mathrm{i}} \equiv B$. Then we set $W / r_{\mathrm{i}}$ $\equiv \phi$ and $\eta \equiv\left(r-r_{\mathrm{i}}\right) / W(0 \leq \eta \leq 1)$ to normalize the eigenfunction $R_{n}$. Note that $B$ is the Biot number. By substituting $\rho_{n} r_{\mathrm{o}}=(1+\phi) x_{n}$ and $\rho_{n} r=(1+\phi \eta) x_{n}$ into Eq. (6), we obtain: 
T. Meshii, et al., Journal of Thermal Stresses, Vol. 27, No. 3, pp. 253 - 267 (2004. 3).

$$
x_{n}\left[J_{1}\left(x_{n}\right) Y_{1}\left((1+\phi) x_{n}\right)-J_{1}\left((1+\phi) x_{n}\right) Y_{1}\left(x_{n}\right)\right]+B\left[J_{0}\left(x_{n}\right) Y_{1}\left((1+\phi) x_{n}\right)-J_{1}\left((1+\phi) x_{n}\right) Y_{0}\left(x_{n}\right)\right]=0
$$

In a similar way, we substitute the non-dimensional parameters defined above into Eq. (7), and then, by applying Eq.

(9), we have

$$
R_{n}(\eta)=2 \pi^{2} x_{n} \frac{\left\{J_{1}\left((1+\phi) x_{n}\right) Y_{1}\left(x_{n}\right)-J_{1}\left(x_{n}\right) Y_{1}\left((1+\phi) x_{n}\right)\right\}}{-4+\pi^{2}\left(B^{2}+x_{n}^{2}\right)\left\{J_{1}\left((1+\phi) x_{n}\right) Y_{0}\left(x_{n}\right)-J_{0}\left(x_{n}\right) Y_{1}\left((1+\phi) x_{n}\right)\right\}^{2}} \times\left\{J_{1}\left((1+\phi) x_{n}\right) Y_{0}\left((1+\phi \eta) x_{n}\right)-J_{0}\left((1+\phi \eta) x_{n}\right) Y_{1}\left((1+\phi) x_{n}\right)\right\}
$$

From Eqs. (9) and (10), we see that

- non-dimensional eigenvalue $x_{n}$ is obtained once non-dimensional parameters $\phi$ (structure) and $B$ (heat transfer) are specified

- since $\phi=1 /\left(r_{\mathrm{m}} / W-1 / 2\right), x_{n}$ is obtained once $r_{\mathrm{m}} / W$ and $B$ are specified, and therefore the eigenfunction can be normalized as a function in a form of $R_{n}(\eta)(0 \leq \eta \leq 1)$.

Subsequently, we normalize the terms related to time in Eq. (8). Here we introduce reference frequency $f^{*} \equiv \kappa / r_{\mathrm{i}}^{2}$, non-dimensional angular velocity $\Omega \equiv \omega /\left(2 \pi f^{*}\right)$ and Fourier number $F_{\mathrm{o}} \equiv f^{*} t$ and then obtain

$$
\begin{gathered}
\omega t=\left(2 \pi f^{*} \Omega\right) \cdot\left(F_{\mathrm{o}} / f^{*}\right)=2 \pi \Omega F_{\mathrm{o}} \\
\frac{\kappa \rho_{n}^{2}}{\omega}=\frac{\kappa \rho_{n}^{2}}{2 \pi f^{*} \Omega}=\frac{\kappa \rho_{n}^{2}}{2 \pi\left(\kappa / r_{\mathrm{i}}^{2}\right) \Omega}=\frac{\left(\rho_{n} r_{\mathrm{i}}\right)^{2}}{2 \pi \Omega}=\frac{x_{n}^{2}}{2 \pi \Omega} \\
\kappa \rho_{n}^{2} t=\kappa \rho_{n}^{2} \frac{F_{\mathrm{o}}}{f^{*}}=\kappa \rho_{n}^{2} \frac{F_{\mathrm{o}}}{\kappa / r_{\mathrm{i}}^{2}}=\left(\rho_{n} r_{\mathrm{i}}\right)^{2} F_{\mathrm{o}}=x_{n}^{2} F_{\mathrm{o}}
\end{gathered}
$$

By substituting equations (11) to (13) into (8), we finally normalized the desired $u$ as follows:

$$
\frac{u\left(\eta, F_{\mathrm{o}}\right)}{\Delta T}=\sin \left(2 \pi \Omega F_{\mathrm{o}}\right)+\sum_{n=1}^{\infty} \frac{R_{n}(\eta)}{1+\left\{x_{n}^{2} /(2 \pi \Omega)\right\}^{2}} \times\left\{\frac{x_{n}^{2}}{2 \pi \Omega}\left(e^{-x_{n}^{2} F_{\mathrm{o}}}-\cos \left(2 \pi \Omega F_{\mathrm{o}}\right)\right)-\sin \left(2 \pi \Omega F_{\mathrm{o}}\right)\right\}
$$

where the non-dimensional parameters related to time are $F_{\mathrm{o}}$ and $\Omega$.

Thus we conclude that once we specify the non-dimensional parameters on structure $r_{\mathrm{m}} / W$, heat transfer $B$ and fluid temperature fluctuation $\Omega$, cylinder temperature change $u$ at non-dimensional time $F_{\mathrm{o}}$ and location $\eta$ can be obtained.

\section{Characteristics of the Eigenvalues}

Through tentative calculations, we know that the eigenvalue $x_{n}$ has the following relationship:

$$
x_{n+1}-x_{n} \approx \frac{\pi}{\phi}=\pi\left(\frac{r_{\mathrm{m}}}{W}-\frac{1}{2}\right)
$$

This relationship is expected from the equation 
T. Meshii, et al., Journal of Thermal Stresses, Vol. 27, No. 3, pp. 253 - 267 (2004. 3).

$$
\frac{2}{\pi \sqrt{1+\phi}}\left[-\frac{B}{x_{n}} \cos \phi x_{n}+\sin \phi x_{n}\right] \approx 0
$$

which is obtained by setting $X=x_{n}$ and substituting the following into Eq. (9):

$$
\begin{gathered}
J_{m}(X) \approx \sqrt{\frac{2}{\pi X}} \cos \left(X-\frac{(2 m+1) \pi}{4}\right) \\
Y_{m}(X) \approx \sqrt{\frac{2}{\pi X}} \sin \left(X-\frac{(2 m+1) \pi}{4}\right)
\end{gathered}
$$

where Eqs. (17) and (18) are integer order Bessel functions' principal asymptotic forms applicable for large $X$ [11].

We see from Eq. (16) that because $x_{n}$ is large, $\sin \phi x_{n} \approx 0$ and thus Eq. (15) is expected. Our experience shows that Eq. (15) is valid for $x_{n}>5$ and that the error in Eq. (15) for this range is less than a few percents.

The characteristic shown above will be useful for numerically obtaining eigenvalues $x_{n}$ by solving Eq. (9), for example, by Newton's method. However, selecting an appropriate trial value for $x_{1}$ is also important in solving Eq. (9) numerically, to collect all the eigenvalues. From our past experience, we had successful results by setting $x_{1}=$ $\pi /(2 \phi)$ and then solving the Eq. (9). Subsequently, the accurately obtained $x_{1}$ is used to set the trial $x_{2}$ by applying Eq. (15) and then the accurate $x_{2}$ is evaluated. This process is repeated till all the necessary eigenvalues are obtained.

The trial value $x_{1}=\pi /(2 \phi)$ is obtained from Eq. (16) in the case $n=1$ and $x_{1} / B<<1$; considering $\cos \phi x_{1} \approx 0$ is necessary in this case. In the cases $x_{1} / B<<1$ is not satisfied, it seems that the trial value $x_{1}=\pi /(2 \phi)$ and the process for solving Eq. (9) shown above is still useful, if we are aware that $x_{1}$ is the smallest eigenvalue and if we always check that the obtained $x_{n}$ is actually the $n$th eigenvalue by applying Eq. (15).

\section{NORMALIZED SIF OF A CIRCUMFERENTIAL CRACK IN THIN- TO THICK-WALLED CYLINDER UNDER THERMAL STRIPING}

In the following, we consider a problem of a hollow cylinder of uniform temperature $T_{\mathrm{m}}(t<0)$, which is insulated adiabatically outside and heated inside by a fluid of $T_{\mathrm{m}}+T_{\mathrm{f}}(t)=T_{\mathrm{m}}+\Delta T \sin \omega t(t \geq 0)$ with heat transfer coefficient $h$. Once the temperature distribution $T_{\mathrm{m}}+u(r, t)$ is obtained at a certain time by applying Eq. (14), the SIF of a circumferential crack in a finite-length cylinder, $K_{\text {cyl }}$, can be obtained by our semi-analytical numerical method [6]. The crack is located at the midpoint of the cylinder length $H$ and the edges of the cylinder are 
rotation-restrained. The edges can move freely in the axial direction, as encountered in practical problems. The material of the cylinder is assumed to be homogeneous with isotropic and temperature independent properties. Bernoulli-Euler assumption that sections which are plane and perpendicular to the axis before loading remains so after loading is applied. The SIF obtained by the method is in the range of less than $5 \%$ difference from that by FEA for a thin- to thick-walled cylinder of $r_{\mathrm{m}} / W \geq 1, \beta H \geq 2.5\left(\beta=\left\{3\left(1-v^{2}\right)\right\}^{1 / 4} /\left(r_{\mathrm{m}} W\right)^{1 / 2}\right.$ is a quantity used in replacing cylindrical shell by a beam on an elastic foundation, where $v$ is the Poisson's ratio) and for a crack depth of $a / W \leq 0.5$ and less than $10 \%$ for $a / W \leq 0.7[12]$.

In concrete, the desired transient SIF $K_{\text {cyl }}$ at a time $t$ is obtained by substituting $u(r, t)$ given by Eq. (14) to the following equations.

$$
\begin{gathered}
K_{\mathrm{cyl}}(t)=K_{\text {freec }}(t)+K_{\mathrm{fbr}}(t) \\
K_{\text {freec }}(t)=\int_{r_{\mathrm{i}}}^{r_{\mathrm{i}}+a} \frac{r\left[-\sigma_{M}(r, t)\right]}{r_{\mathrm{i}}+a} \cdot w\left(r-r_{\mathrm{i}} ; a\right) d r \\
K_{\text {fbr }}(t)=F_{\mathrm{ttbr}} \cdot\left\{\frac{-6 M_{\mathrm{t}}(t)}{W^{2}} \sqrt{\pi a} \cdot F_{M}(a / W)\right\} \\
\sigma_{M}(r, t)=\frac{E \alpha}{1-v}\left(u(r, t)-u_{\mathrm{avg}}(t)\right) \\
u_{\text {avg }}(t)=\left\{\int_{r_{\mathrm{i}}}^{r_{\mathrm{o}}} 2 \pi r \cdot u(r, t) d r\right\} /\left\{\pi\left(r_{\mathrm{o}}^{2}-r_{\mathrm{i}}^{2}\right)\right\} \\
M_{\mathrm{t}}(t)=\int_{r_{\mathrm{i}}}^{r_{\mathrm{o}}} \sigma_{M} \cdot\left(r_{\mathrm{m}}-r\right) d r
\end{gathered}
$$

where $E, v$ and $\alpha$ are Young's modulus, Poisson's ratio and coefficient of thermal expansion. $F_{M}$ is the correction factor of finite width for single edge cracked strip under pure bending. $W$ and $F_{\text {tfbr }}$ are functions of structural parameters for a circumferential crack, given concretely in the reference [6].

\section{Normalization of SIF for a Circumferential Crack under Thermal Striping}

We first show that the SIF $K_{\text {cyl }}$ is identical for various materials, cylinder configurations, heat transfer conditions and thermal striping frequency, if all of the non-dimensional parameters which were discussed in the previous section are set at the same, respectively. The reference case is as follows: austenitic stainless cylinder (specified as SUS in the following) of $r_{\mathrm{m}} / W=10.5, H=\pi / \beta, W=10 \mathrm{~mm}, a / W=0.5, E=198 \mathrm{GPa}, \alpha=1.6 \times 10^{-5} \mathrm{~K}^{-1}, v=0.3, \Lambda$ 
T. Meshii, et al., Journal of Thermal Stresses, Vol. 27, No. 3, pp. 253 - 267 (2004. 3).

$=12.677 \mathrm{~W} / \mathrm{mK}, \kappa=3.6111 \mathrm{~mm}^{2} / \mathrm{s}$ and heat transfer coefficient $h=11630 \mathrm{~W} / \mathrm{m}^{2} \mathrm{~K}(B=91.74)$ and thermal striping of $\Delta T=50 \mathrm{~K}, f=0.05 \mathrm{~Hz}(\Omega=138.5)$. We chose the cylinder length $H$ as specified because we know that the SIF for the problem takes approximately the maximum value near this $H[6]$.

The transient SIF $K_{\text {cyl }}$ for this reference case was calculated for various $t$ in the range of $t=999 / f \sim 1000 / f$ ( $1000^{\text {th }}$ cycle) by substituting $u(r, t)$ obtained by Eq. (14) into Eqs. (19) $\sim(24)$. The results showed that the $K_{\text {cyl }}$ takes the minimum and maximum values at $t=19985.5$ and $19995.5 \mathrm{sec}\left(F_{\mathrm{o}}=7.217,7.221\right)$, respectively. We compare this peak SIFs of the reference cases with the SIFs for the following four cases i) $\sim$ iv), whose $h, f$ and $t$ were chosen to make the non-dimensional parameters as same as the reference case. All the calculated SIFs were normalized by the $K_{\Delta T}$ as defined below and summarized in table 1 . Note that the negative $K_{\text {cyl }}$ in Table 1 just represent decrement in SIF from the steady state value, and does not necessarily mean that the total SIF becomes negative.

$$
K_{\Delta T}=\frac{E \alpha \Delta T}{1-v} \sqrt{\pi W}
$$

i) A case where material constants are identical with the reference case and $W=100 \mathrm{~mm}$

ii) A case where material constants are chosen for Ti-6Al-4V ( $\kappa, \Lambda$ is very small compared with other alloys) [13] and $W=10 \mathrm{~mm}$

iii) A case where material constants are chosen for $\mathrm{Cu}(\mathrm{C} 16200 ; \kappa, \Lambda$ is very large compared with other alloys) [13] and $W=10 \mathrm{~mm}$

iv) A case where material constants are chosen for Ti-6Al-4V ( $\kappa, \Lambda$ is very small compared with other alloys) [13] and $W=100 \mathrm{~mm}$

We see from table 1 that the normalized transient SIF $K_{\mathrm{cyl}} / K_{\Delta T}$ at non-dimensional time $F_{\mathrm{o}}$ is identical when the values of the non-dimensional quantities $r_{\mathrm{m}} / W, H / W, a / W, B$ and $\Omega$ are set to take the same values, respectively, regardless of materials nor structures.

\section{Normalized SIF Range of a Circumferential Crack under Thermal Striping}

Finally we presented the normalized SIF range $\Delta K_{\text {cyl }} / K_{\Delta T}$ for the first cycle and steady state (described as $\infty$ th 
T. Meshii, et al., Journal of Thermal Stresses, Vol. 27, No. 3, pp. 253 - 267 (2004. 3).

cycle in the tables) for various non-dimensional structural, heat transfer and thermal striping parameters $r_{\mathrm{m}} / W, a / W$, $B$ and $\Omega$, respectively, considering the fact that the SIF solutions will be applied in the fatigue crack propagation with the Paris law [7]. The results for $r_{\mathrm{m}} / W=10,5$ and 1 are presented in tables 2, 3 and 4, respectively. In these tables, we again chose the cylinder length $H=\pi / \beta$ because we know that the SIF for the problem takes approximately the maximum value near this $H$ [6]. In addition, this knowledge on the effect of cylinder length on the SIF was checked for the cases in tables 2 to 4 by recalculating the SIF ranges for $H=5 / \beta$ (which is known to satisfy the long cylinder condition [6]) and summarized as tables 5 to 7 , respectively. For all cases, Poisson's ratio was set as $v=0.3$.

We see from the tables that

- $\Delta K_{\text {cyl }} / K_{\Delta T}$ at the $1^{\text {st }}$ cycle is not necessarily larger than that for steady state $\Delta K_{\text {cyl }} / K_{\Delta T}$, for a specified $r_{\mathrm{m}} / W, H / W$, $a / W, B$ and $\Omega$. This tendency becomes more apparent for small $\Omega$ and large $r_{\mathrm{m}} / W$.

- The $\Omega$ for which $\Delta K_{\text {cyl }} / K_{\Delta T}$ takes a maximum might vary by a selection in pair of $r_{\mathrm{m}} / W$ and $B$. From the results in the tables, this $\Omega$ is 100 or 10 for most cases.

- For a set of $r_{\mathrm{m}} / W, H / W, B$ and $\Omega, \Delta K_{\text {cyl }} / K_{\Delta T}$ decreases (in an engineering sense) for the increase in $a / W$. There are cases that $\Delta K_{\mathrm{cyl}} / K_{\Delta T}$ increase for intermediate $a / W$, but will finally decrease for large $a / W$. This suggests that the fatigue crack growth rate under thermal striping, for crack in consideration, will show the decrease tendency when the crack becomes deep. This tendency is favorable.

- For a set of $r_{\mathrm{m}} / W, a / W, B$ and $\Omega, \Delta K_{\text {cyl }} / K_{\Delta T}$ for $H=\pi / \beta$ is generally not less than that for $H=5 / \beta$. For example, the difference in $\Delta K_{\text {cyl }} / K_{\Delta T}$ when $r_{\mathrm{m}} / W=10$ and 5 is approximately $0.1 \%$ for $a / W=0.05$ and $10 \%$ for $a / W=0.7$. When we remember that $\Delta K_{\text {cyl }} / K_{\Delta T}$ was also the maximum for $H=\pi / \beta$ for step temperature change [6] and that the transient SIF is dominated by structural parameters for the step temperature change [12], we think that transient SIF range under thermal striping in short (such as short pipes between elbows and nozzles in a pressure vessel) to long cylinders can be evaluated conservatively by the SIF range for $H=\pi / \beta$ given in tables 2 to 4 . 


\section{CONCLUSIONS}

In this paper we considered the normalized SIF range of an inner-surface circumferential crack in a thin- to thick-walled finite-length cylinder under thermal striping. For this purpose, we rewrote our analytical transient temperature solution of the cylinder under thermal striping. Then we combined this normalized temperature solution for the problem and our semi-analytical numerical SIF evaluation method for the crack, and as a result, showed that the transient SIF solution can be expressed in a generalized form by dimensionless parameters such as mean radius to wall thickness ratio $r_{\mathrm{m}} / W$, Biot number $B=h r_{\mathrm{i}} / \Lambda$, normalized striping frequency $\Omega=f / f^{*}$ and Fourier number $F_{\mathrm{o}}$ $=f^{*} t$. Here, $h$ : heat transfer coefficient, $r_{\mathrm{i}}$ : inner radius, $\Lambda$ : thermal conductivity coefficient, $f:$ thermal striping frequency, $f^{*}=\kappa / r_{\mathrm{i}}{ }^{2}, \kappa$. coefficient of diffusivity and $t$ : time. Finally, considering that our SIF solutions will be applied in fatigue crack growth analysis, normalized SIF ranges for the 1st cycle and steady state were given for these dimensionless parameters in the tables for $r_{\mathrm{m}} / W=10,5$ and 1 .

\section{REFERENCES}

[1] T. Hoshino, T. Aoki, T. Ueno, and Y. Kutomi, Leakage from CVCS Pipe of Regenerative Heat Exchanger Induced by High-Cycle Thermal Fatigue at Tsuruga Nuclear Power Station Unit 2, Proc. ICONE 8, 2000.

[2] A. A. Rizk and F. Erdogan, Cracking of Coated Materials under Transient Thermal Stresses, Journal of Thermal Stresses, Vol. 12, pp. 125-168, 1989.

[3] F. Erdogan and A. A. Rizk, Fracture of Coated Plates and Shells under Thermal Shock, International Journal of Fracture, Vol. 53, pp.159-185, 1992.

[4] H. F. Nied and F. Erdogan, The Elasticity Problem for a Thick-Walled Cylinder Containing a Circumferential Crack, International Journal of Fracture, Vol. 22, pp.277-301, 1983.

[5] H. F. Nied and F. Erdogan, Transient Thermal Stress Problem for a Circumferentially Cracked Hollow Cylinder, Journal of Thermal Stresses, Vol. 6, pp. 1-14, 1983.

[6] T. Meshii and K. Watanabe, Maximum Stress Intensity Factor for a Circumferential Crack in a Finite Length 
T. Meshii, et al., Journal of Thermal Stresses, Vol. 27, No. 3, pp. 253 - 267 (2004. 3).

Thin-Walled Cylinder under Transient Radial Temperature Distribution, Engineering Fracture Mechanics, Vol. 63, pp. 23-38, 1999.

[7] P. C. Paris and F. Erdogan, A Critical Analysis of Crack Propagation Laws, Transactions of ASME Ser. D, Vol. 85 , pp. 528-534, 1963.

[8] T. Meshii and K. Watanabe, 2002, "Stress Intensity Factor of a Circumferential Crack in a Thick-Walled Cylinder under Thermal Striping,” Proc. 2002 ASME Pressure Vessels and Piping Conference, Vol. 443-1, p. 93, 2002.

[9] H. S. Carslaw and J. C. Jaeger, Conduction of Heat in Solids, 2nd ed., Oxford University Press, Oxford, 1946.

[10] S. J. Farlow, Partial Differential Equations for Scientists and Engineers, John Wiley \& Sons, New York, 1982.

[11] M. Abramowitz and I. A. Stegun, Handbook of Mathematical Functions, 9th printing, Dover Publications, New York, 1972.

[12] T. Meshii and K. Watanabe, Crack Arrest Analysis under Cyclic Thermal Shock for an Inner-Surface Circumferential Crack in a Finite-Length Thick-Walled Cylinder, J. Thermal Stresses, Vol. 25, pp. 1121-1132, 2002.

[13] Japan Society of Mechanical Engineers, JSME Data Book: Heat Transfer, $4^{\text {th }}$ ed., JSME, Tokyo, 1986. 
T. Meshii, et al., Journal of Thermal Stresses, Vol. 27, No. 3, pp. 253 - 267 (2004. 3).

\section{List of figures and tables}

Figure 1 Circumferentially cracked cylinder under radial temperature distribution.

Table 1 Normalized transient stress intensity factor $K_{\mathrm{cy}} / K_{\Delta T}\left(r_{\mathrm{m}} / W=10.5, H=\pi / \beta, a / W=0.5, B=91.74, \Omega=138.5\right.$, $\nu=0.3)$.

Table 2 Normalized transient stress intensity factor range $\Delta K_{\text {cyl }} / K_{\Delta T}$ at $1^{\text {st }}$ and $\infty$ cycle $\left(r_{\mathrm{m}} / W=10, H=\pi / \beta, v=0.3\right)$.

Table 3 Normalized transient stress intensity factor range $\Delta K_{\text {cyl }} / K_{\Delta T}$ at $1^{\text {st }}$ and $\infty$ cycle $\left(r_{\mathrm{m}} / W=5, H=\pi / \beta, v=0.3\right)$.

Table 4 Normalized transient stress intensity factor range $\Delta K_{\text {cyl }} / K_{\Delta T}$ at $1^{\text {st }}$ and $\infty$ cycle $\left(r_{\mathrm{m}} / W=1, H=\pi / \beta, v=0.3\right)$.

Table 5 Normalized transient stress intensity factor range $\Delta K_{\text {cyl }} / K_{\Delta T}$ at $1^{\text {st }}$ and $\infty$ cycle $\left(r_{\mathrm{m}} / W=10, H=5 / \beta, \nu=0.3\right)$.

Table 6 Normalized transient stress intensity factor range $\Delta K_{\text {cyl }} / K_{\Delta T}$ at $1^{\text {st }}$ and $\infty$ cycle $\left(r_{\mathrm{m}} / W=5, H=5 / \beta, v=0.3\right)$.

Table 7 Normalized transient stress intensity factor range $\Delta K_{\text {cyl }} / K_{\Delta T}$ at $1^{\text {st }}$ and $\infty$ cycle $\left(r_{\mathrm{m}} / W=1, H=5 / \beta, v=0.3\right)$. 
T. Meshii, et al., Journal of Thermal Stresses, Vol. 27, No. 3, pp. 253 - 267 (2004. 3).

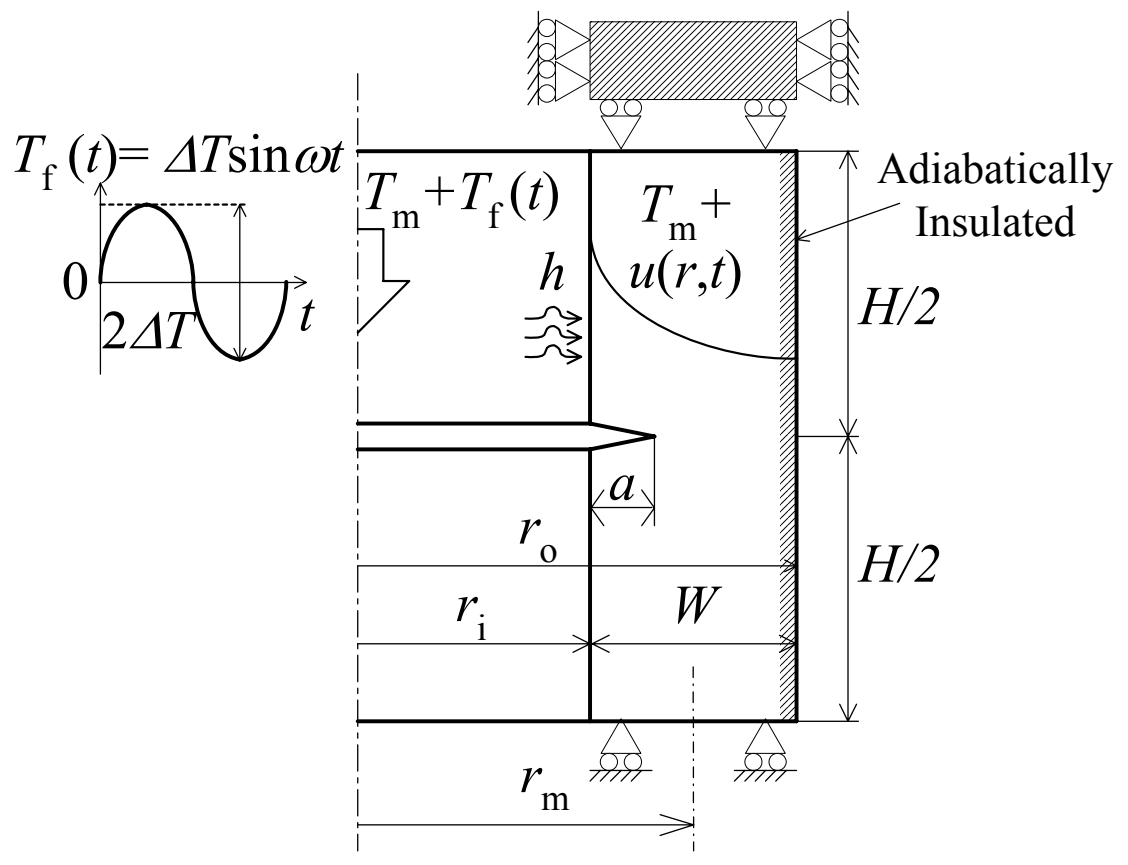

Figure 1 Circumferentially cracked cylinder under radial temperature distribution.

Table 1 Normalized transient stress intensity factor $K_{\text {cyl }} / K_{\Delta T}$

$$
\left(r_{\mathrm{m}} / W=10.5, H=\pi / \beta, a / W=0.5, B=91.74, \Omega=138.5, v=0.3\right) .
$$

\begin{tabular}{llllrrrrrr}
\hline$F_{\mathrm{o}}$ & $r_{\mathrm{i}}$ & $W$ & Material & \multicolumn{1}{c}{$\begin{array}{c}\kappa \\
\mathrm{W} / \mathrm{mK}\end{array}$} & \multicolumn{1}{c}{\begin{tabular}{c}
\multicolumn{1}{c}{$\begin{array}{c}\mathrm{mm}^{2} / \mathrm{s} \\
\mathrm{m} / \mathrm{m}^{2} \mathrm{~K}\end{array}$} \\
\end{tabular}} & \multicolumn{1}{l}{$\mathrm{m}$} & \multicolumn{1}{c}{$\mathrm{Hz}$} & \multicolumn{1}{c}{$\mathrm{s}$} & $K_{\text {cyl }} / K_{\Delta T}$ \\
\hline 7.217 & 0.100 & 0.010 & SUS & 12.677 & 3.6111 & 11630 & 0.0500 & 19985.5 & -0.276 \\
& 1.000 & 0.100 & SUS & 12.677 & 3.6111 & 1163 & 0.00050 & 1998550 & -0.276 \\
& 0.100 & 0.010 & Ti-6Al-4V & 7.60 & 2.13 & 6972 & 0.0295 & 33882.6 & -0.276 \\
& 0.100 & 0.010 & Cu & 360 & 106.60 & 330275 & 1.476 & 677.0 & -0.276 \\
& 1.000 & 0.100 & Ti-6Al-4V & 7.60 & 2.13 & 697.2 & 0.000295 & 3388256 & -0.276 \\
\hline 7.221 & 0.100 & 0.010 & SUS & 12.677 & 3.6111 & 11630 & 0.0500 & 19995.5 & 0.276 \\
& 1.000 & 0.100 & SUS & 12.677 & 3.6111 & 1163 & 0.00050 & 1999550 & 0.276 \\
& 0.100 & 0.010 & Ti-6Al-4V & 7.60 & 2.13 & 6972 & 0.0295 & 33899.5 & 0.276 \\
& 0.100 & 0.010 & Cu & 360 & 106.60 & 330275 & 1.476 & 677.4 & 0.276 \\
& 1.000 & 0.100 & Ti-6Al-4V & 7.60 & 2.13 & 697.2 & 0.000295 & 3389952 & 0.276 \\
\hline
\end{tabular}


T. Meshii, et al., Journal of Thermal Stresses, Vol. 27, No. 3, pp. 253 - 267 (2004. 3).

Table 2 Normalized transient stress intensity factor range $\Delta K_{\mathrm{cyl}} / K_{\Delta T}$ at $1^{\text {st }}$ and $\infty$ cycle $\left(r_{\mathrm{m}} / W=10, H=\pi / \beta, \nu=0.3\right)$.

\begin{tabular}{|c|c|c|c|c|c|c|c|c|c|c|}
\hline \multirow[b]{2}{*}{$B$} & \multirow[b]{2}{*}{$\Omega$} & \multirow[b]{2}{*}{ cycle } & \multicolumn{7}{|c|}{$a / W$} & \multirow[b]{2}{*}{0.7} \\
\hline & & & 0.05 & 0.1 & 0.2 & 0.3 & 0.4 & 0.5 & 0.6 & \\
\hline \multirow[t]{8}{*}{10000} & \begin{tabular}{|l|l}
10000 \\
\end{tabular} & & 0.277 & 0.226 & 0.137 & 0.119 & 0.121 & 0.123 & 0.121 & 0.126 \\
\hline & & $\infty$ & 0.270 & 0.213 & 0.126 & 0.114 & 0.115 & 0.116 & 0.114 & 0.119 \\
\hline & 1000 & 1 & 0.382 & 0.447 & 0.445 & 0.410 & 0.384 & 0.366 & 0.344 & 0.342 \\
\hline & & $\infty$ & 0.382 & 0.443 & 0.432 & 0.390 & 0.359 & 0.339 & 0.319 & 0.321 \\
\hline & 100 & 1 & 0.344 & 0.447 & 0.541 & 0.590 & 0.626 & 0.640 & 0.613 & 0.569 \\
\hline & & $\infty$ & 0.369 & 0.479 & 0.581 & 0.634 & 0.673 & 0.688 & 0.659 & 0.612 \\
\hline & 10 & 1 & 0.101 & 0.131 & 0.161 & 0.177 & 0.189 & 0.195 & 0.188 & 0.174 \\
\hline & & $\infty$ & 0.110 & 0.144 & 0.176 & 0.194 & 0.207 & 0.213 & 0.205 & 0.190 \\
\hline \multirow[t]{8}{*}{1000} & $\begin{array}{|ll|}10000 \\
\end{array}$ & 1 & 0.239 & 0.195 & 0.118 & 0.103 & 0.104 & 0.105 & 0.104 & 0.108 \\
\hline & & $\infty$ & 0.231 & 0.182 & 0.108 & 0.097 & 0.098 & 0.099 & 0.098 & 0.102 \\
\hline & 1000 & 1 & 0.364 & 0.426 & 0.424 & 0.391 & 0.366 & 0.349 & 0.328 & 0.326 \\
\hline & & $\infty$ & 0.363 & 0.421 & 0.411 & 0.370 & 0.341 & 0.322 & 0.303 & 0.305 \\
\hline & 100 & 1 & 0.339 & 0.439 & 0.533 & 0.580 & 0.616 & 0.630 & 0.603 & 0.560 \\
\hline & & $\infty$ & 0.363 & 0.471 & 0.571 & 0.623 & 0.661 & 0.676 & 0.647 & 0.601 \\
\hline & 10 & 1 & 0.100 & 0.131 & 0.161 & 0.177 & 0.189 & 0.194 & 0.187 & 0.174 \\
\hline & & $\infty$ & 0.110 & 0.143 & 0.176 & 0.194 & 0.207 & 0.213 & 0.204 & 0.190 \\
\hline \multirow[t]{8}{*}{100} & 10000 & 1 & 0.090 & 0.073 & 0.043 & 0.037 & 0.038 & 0.039 & 0.038 & 0.040 \\
\hline & & $\infty$ & 0.083 & 0.066 & 0.039 & 0.035 & 0.035 & 0.036 & 0.035 & 0.037 \\
\hline & 1000 & 1 & 0.237 & 0.278 & 0.277 & 0.256 & 0.239 & 0.228 & 0.214 & 0.212 \\
\hline & & $\infty$ & 0.231 & 0.268 & 0.261 & 0.236 & 0.217 & 0.205 & 0.193 & 0.194 \\
\hline & 100 & 1 & 0.289 & 0.375 & 0.455 & 0.496 & 0.526 & 0.539 & 0.516 & 0.479 \\
\hline & & $\infty$ & 0.306 & 0.397 & 0.482 & 0.526 & 0.558 & 0.571 & 0.546 & 0.508 \\
\hline & 10 & 1 & 0.098 & 0.127 & 0.156 & 0.172 & 0.184 & 0.189 & 0.182 & 0.169 \\
\hline & & $\infty$ & 0.108 & 0.141 & 0.173 & 0.191 & 0.204 & 0.209 & 0.201 & 0.187 \\
\hline \multirow[t]{8}{*}{10} & 10000 & 1 & 0.011 & 0.009 & 0.005 & 0.005 & 0.005 & 0.005 & 0.005 & 0.005 \\
\hline & & $\infty$ & 0.011 & 0.008 & 0.005 & 0.004 & 0.005 & 0.005 & 0.005 & 0.005 \\
\hline & 1000 & 1 & 0.047 & 0.055 & 0.055 & 0.050 & 0.047 & 0.044 & 0.042 & 0.041 \\
\hline & & $\infty$ & 0.044 & 0.051 & 0.050 & 0.045 & 0.041 & 0.039 & 0.037 & 0.037 \\
\hline & 100 & 1 & 0.107 & 0.138 & 0.168 & 0.183 & 0.194 & 0.199 & 0.190 & 0.177 \\
\hline & & $\infty$ & 0.107 & 0.139 & 0.169 & 0.184 & 0.196 & 0.200 & 0.191 & 0.178 \\
\hline & 10 & 1 & 0.072 & 0.094 & 0.115 & 0.126 & 0.135 & 0.139 & 0.134 & 0.124 \\
\hline & & $\infty$ & 0.083 & 0.108 & 0.133 & 0.146 & 0.156 & 0.161 & 0.154 & 0.143 \\
\hline
\end{tabular}

Table 3 Normalized transient stress intensity factor range $\Delta K_{\text {cyl }} / K_{\Delta T}$ at $1^{\text {st }}$ and $\infty$ cycle $\left(r_{\mathrm{m}} / W=5, H=\pi / \beta, v=0.3\right)$.

\begin{tabular}{|c|c|c|c|c|c|c|c|c|c|c|}
\hline \multirow[b]{2}{*}{$B$} & \multirow[b]{2}{*}{$\Omega$} & \multirow[b]{2}{*}{ cycle } & \multicolumn{7}{|c|}{$a / W$} & \multirow[b]{2}{*}{0.7} \\
\hline & & & 0.05 & 0.1 & 0.2 & 0.3 & 0.4 & 0.5 & 0.6 & \\
\hline \multirow[t]{8}{*}{10000} & 10000 & 1 & 0.155 & 0.090 & 0.060 & 0.052 & 0.049 & 0.046 & 0.042 & 0.041 \\
\hline & & $\infty$ & 0.146 & 0.083 & 0.058 & 0.049 & 0.046 & 0.043 & 0.039 & 0.039 \\
\hline & 1000 & 1 & 0.331 & 0.317 & 0.218 & 0.162 & 0.146 & 0.138 & 0.125 & 0.122 \\
\hline & & $\infty$ & 0.326 & 0.305 & 0.201 & 0.149 & 0.138 & 0.131 & 0.119 & 0.116 \\
\hline & 100 & 1 & 0.389 & 0.477 & 0.513 & 0.496 & 0.467 & 0.426 & 0.364 & 0.313 \\
\hline & & $\infty$ & 0.392 & 0.478 & 0.509 & 0.486 & 0.452 & 0.408 & 0.346 & 0.299 \\
\hline & 10 & 1 & 0.277 & 0.358 & 0.426 & 0.449 & 0.454 & 0.437 & 0.387 & 0.326 \\
\hline & & $\infty$ & 0.316 & 0.409 & 0.487 & 0.513 & 0.519 & 0.499 & 0.442 & 0.371 \\
\hline \multirow[t]{8}{*}{1000} & 10000 & 1 & 0.134 & 0.077 & 0.052 & 0.045 & 0.042 & 0.039 & 0.036 & 0.035 \\
\hline & & $\infty$ & 0.125 & 0.071 & 0.049 & 0.042 & 0.039 & 0.037 & 0.034 & 0.033 \\
\hline & 1000 & 1 & 0.315 & 0.302 & 0.207 & 0.154 & 0.139 & 0.131 & 0.119 & 0.116 \\
\hline & & $\infty$ & 0.309 & 0.290 & 0.191 & 0.141 & 0.131 & 0.125 & 0.113 & 0.110 \\
\hline & 100 & 1 & 0.383 & 0.469 & 0.506 & $\overline{0.4}$ & $\overline{0.4}$ & & 359 & 0.309 \\
\hline & & $\infty$ & 0.386 & 0.470 & 0.500 & 0.478 & 0.445 & 0.401 & 0.341 & 0.294 \\
\hline & 10 & 1 & 0.276 & 0.356 & 0.424 & 0.446 & 0.452 & 0.435 & 0.385 & 0.324 \\
\hline & & $\infty$ & 0.314 & 0.406 & 0.484 & 0.510 & 0.516 & 0.497 & 0.439 & 0.369 \\
\hline \multirow[t]{8}{*}{100} & 10000 & 1 & 0.050 & 0.028 & 0.019 & 0.016 & 0.015 & 0.014 & 0.013 & 0.013 \\
\hline & & $\infty$ & 0.045 & 0.026 & 0.018 & 0.015 & 0.014 & 0.013 & 0.012 & 0.012 \\
\hline & 1000 & 1 & 0.205 & 0.197 & 0.135 & 0.100 & 0.090 & 0.085 & 0.077 & 0.075 \\
\hline & & $\infty$ & 0.197 & 0.185 & & 0.090 & & 0.07 & 0.072 & 0.070 \\
\hline & 100 & 1 & 0.329 & 0.403 & 0.4 & 0.420 & & $\overline{0.3}$ & 0.309 & 0.266 \\
\hline & & $\infty$ & 0.329 & 0.400 & 0.42 & 0.407 & & 0.342 & 0.290 & 0.250 \\
\hline & 10 & 1 & 0.262 & 0.338 & 0.402 & 0.424 & 0.429 & 0.413 & 0.366 & 0.308 \\
\hline & & $\infty$ & 0.297 & 0.384 & 0.457 & 0.481 & 0.487 & 0.469 & 0.415 & 0.349 \\
\hline \multirow[t]{8}{*}{10} & 10000 & 1 & 0.006 & 0.003 & 0.002 & 0.002 & 0.002 & 0.002 & 0.002 & 0.002 \\
\hline & & $\infty$ & 0.006 & 0.003 & 0.002 & 0.002 & 0.002 & 0.002 & 0.002 & 0.002 \\
\hline & 1000 & 1 & 0.040 & 0.039 & 0.026 & 0.019 & 0.017 & 0.016 & 0.015 & 0.014 \\
\hline & & $\infty$ & 0.038 & 0.035 & 0.023 & 0.017 & 0.016 & 0.015 & 0.014 & 0.013 \\
\hline & 100 & 1 & 0.123 & 0.152 & 0.164 & 0.159 & 0.150 & 0.137 & 0.117 & 0.100 \\
\hline & & $\infty$ & 0.118 & 0.144 & 0.153 & 0.146 & 0.136 & 0.123 & 0.104 & 0.090 \\
\hline & 10 & & 0.165 & 0.213 & 0.253 & 0.267 & & 0.260 & 0.230 & 0.193 \\
\hline & & & 0.178 & 0.229 & 0.273 & 0.288 & 0.291 & 0.280 & 0.248 & 0.20 \\
\hline
\end{tabular}


T. Meshii, et al., Journal of Thermal Stresses, Vol. 27, No. 3, pp. 253 - 267 (2004. 3).

Table 4 Normalized transient stress intensity factor range $\Delta K_{\text {cyl }} / K_{\Delta T}$ at $1^{\text {st }}$ and $\infty$ cycle $\left(r_{\mathrm{m}} / W=1, H=\pi / \beta, v=0.3\right)$.

\begin{tabular}{|c|c|c|c|c|c|c|c|c|c|c|}
\hline \multirow[b]{2}{*}{$B$} & \multirow[b]{2}{*}{$\Omega$} & \multirow[b]{2}{*}{ cycle } & \multicolumn{7}{|c|}{$a / W$} & \multirow[b]{2}{*}{0.7} \\
\hline & & & 0.05 & 0.1 & 0.2 & 0.3 & 0.4 & 0.5 & 0.6 & \\
\hline \multirow[t]{8}{*}{10000} & \begin{tabular}{|l|}
10000 \\
\end{tabular} & & 0.016 & 0.009 & 0.005 & 0.003 & 0.002 & 0.002 & 0.001 & 0.001 \\
\hline & & $\infty$ & 0.015 & 0.009 & 0.004 & 0.003 & 0.002 & 0.002 & 0.001 & 0.000 \\
\hline & 1000 & 1 & 0.042 & 0.027 & 0.014 & 0.009 & 0.006 & 0.004 & 0.002 & 0.002 \\
\hline & & $\infty$ & 0.040 & 0.025 & 0.013 & 0.008 & 0.006 & 0.004 & 0.002 & 0.002 \\
\hline & 100 & 1 & 0.166 & 0.089 & 0.045 & 0.028 & 0.019 & 0.013 & 0.008 & 0.006 \\
\hline & & $\infty$ & 0.157 & 0.081 & 0.043 & 0.027 & 0.018 & 0.012 & 0.007 & 0.006 \\
\hline & 10 & 1 & 0.331 & 0.306 & 0.181 & 0.098 & 0.060 & 0.039 & 0.024 & 0.018 \\
\hline & & $\infty$ & 0.326 & 0.296 & 0.168 & 0.088 & 0.055 & 0.038 & 0.023 & 0.017 \\
\hline \multirow[t]{8}{*}{1000} & $\begin{array}{|ll|}10000 \\
\end{array}$ & 1 & 0.013 & 0.008 & 0.004 & 0.003 & 0.002 & 0.001 & 0.001 & 0.000 \\
\hline & & $\infty$ & 0.012 & 0.007 & 0.004 & 0.002 & 0.002 & 0.001 & 0.001 & 0.000 \\
\hline & 1000 & 1 & 0.040 & 0.025 & 0.013 & 0.008 & 0.006 & 0.004 & 0.002 & 0.002 \\
\hline & & $\infty$ & 0.038 & 0.024 & 0.013 & 0.008 & 0.005 & 0.003 & 0.002 & 0.002 \\
\hline & 100 & 1 & 0.164 & 0.088 & 0.044 & 0.028 & 0.019 & 0.012 & 0.007 & 0.006 \\
\hline & & $\infty$ & 0.155 & 0.080 & 0.043 & 0.027 & 0.018 & 0.012 & 0.007 & 0.005 \\
\hline & 10 & 1 & 0.329 & 0.304 & 0.180 & 0.097 & 0.059 & 0.039 & 0.024 & $\overline{0.018}$ \\
\hline & & $\infty$ & 0.324 & 0.294 & 0.167 & 0.088 & 0.055 & 0.037 & 0.023 & 0.017 \\
\hline \multirow[t]{8}{*}{100} & 10000 & 1 & 0.004 & 0.002 & 0.001 & 0.001 & 0.001 & 0.000 & 0.000 & 0.000 \\
\hline & & $\infty$ & 0.004 & 0.002 & 0.001 & 0.001 & 0.001 & 0.000 & 0.000 & 0.000 \\
\hline & 1000 & 1 & 0.026 & 0.016 & 0.009 & 0.005 & $\overline{0.004}$ & 0.002 & 0.001 & 0.001 \\
\hline & & $\infty$ & 0.025 & 0.015 & 0.008 & 0.005 & 0.003 & 0.002 & 0.001 & 0.001 \\
\hline & 100 & 1 & 0.141 & 0.075 & 0.038 & 0.024 & 0.016 & 0.011 & 0.006 & 0.005 \\
\hline & & $\infty$ & 0.132 & 0.068 & 0.036 & 0.023 & 0.015 & 0.010 & 0.006 & 0.005 \\
\hline & 10 & 1 & 0.312 & 0.289 & 0.171 & 0.093 & 0.056 & 0.037 & 0.022 & 0.017 \\
\hline & & $\infty$ & 0.307 & 0.278 & 0.158 & 0.083 & 0.052 & 0.035 & 0.022 & 0.016 \\
\hline \multirow[t]{8}{*}{10} & 10000 & 1 & 0.001 & 0.000 & 0.000 & 0.000 & 0.000 & 0.000 & 0.000 & 0.000 \\
\hline & & $\infty$ & 0.001 & 0.000 & 0.000 & 0.000 & 0.000 & 0.000 & 0.000 & 0.000 \\
\hline & 1000 & 1 & 0.005 & 0.003 & 0.002 & 0.001 & 0.001 & $\overline{0.000}$ & 0.000 & 0.000 \\
\hline & & $\infty$ & 0.005 & 0.003 & 0.002 & 0.001 & 0.001 & 0.000 & 0.000 & 0.000 \\
\hline & 100 & 1 & 0.052 & 0.027 & 0.014 & 0.009 & 0.006 & 0.004 & 0.002 & 0.002 \\
\hline & & $\infty$ & 0.047 & 0.025 & 0.013 & 0.008 & 0.005 & 0.004 & 0.002 & 0.002 \\
\hline & 10 & 1 & 0.199 & 0.184 & 0.109 & 0.058 & 0.035 & 0.023 & 0.014 & 0.011 \\
\hline & & $\infty$ & 0.191 & 0.174 & 0.098 & 0.052 & 0.032 & 0.022 & 0.014 & 0.010 \\
\hline
\end{tabular}

Table 5 Normalized transient stress intensity factor range $\Delta K_{\text {cyl }} / K_{\Delta T}$ at $1^{\text {st }}$ and $\infty$ cycle $\left(r_{\mathrm{m}} / W=10, H=5 / \beta, v=0.3\right)$.

\begin{tabular}{|c|c|c|c|c|c|c|c|c|c|c|}
\hline \multirow[b]{2}{*}{$B$} & \multirow[b]{2}{*}{$\Omega$} & \multirow[b]{2}{*}{ cycle } & \multicolumn{7}{|c|}{$a / W$} & \multirow[b]{2}{*}{0.7} \\
\hline & & & 0.05 & 0.1 & 0.2 & 0.3 & 0.4 & 0.5 & 0.6 & \\
\hline \multirow[t]{8}{*}{10000} & 10000 & 1 & 0.277 & 0.225 & 0.133 & 0.114 & 0.114 & 0.114 & 0.111 & 0.114 \\
\hline & & $\infty$ & 0.270 & 0.211 & 0.122 & 0.108 & 0.108 & 0.108 & 0.105 & 0.108 \\
\hline & 1000 & 1 & 0.382 & 0.445 & 0.438 & 0.397 & 0.366 & 0.343 & 0.318 & 0.308 \\
\hline & & $\infty$ & 0.382 & 0.441 & 0.425 & 0.377 & 0.341 & 0.316 & 0.293 & 0.289 \\
\hline & 100 & 1 & 0.344 & 0.445 & 0.536 & 0.579 & 0.607 & 0.613 & 0.578 & 0.522 \\
\hline & & $\infty$ & 0.369 & 0.478 & 0.576 & 0.622 & 0.653 & 0.659 & 0.622 & 0.561 \\
\hline & 10 & 1 & 0.101 & 0.131 & 0.160 & 0.174 & 0.184 & 0.187 & 0.177 & 0.160 \\
\hline & & $\infty$ & 0.110 & 0.143 & 0.175 & 0.190 & 0.201 & 0.204 & 0.193 & 0.174 \\
\hline \multirow[t]{8}{*}{1000} & 10000 & 1 & 0.239 & 0.194 & 0.115 & 0.098 & 0.098 & 0.098 & 0.096 & 0.098 \\
\hline & & $\infty$ & 0.230 & 0.181 & 0.105 & 0.093 & 0.092 & 0.092 & 0.090 & 0.092 \\
\hline & $\begin{array}{l}1000 \\
\end{array}$ & 1 & 0.364 & 0.424 & 0.418 & 0.379 & 0.349 & 0.327 & 0.303 & 0.294 \\
\hline & & $\infty$ & 0.363 & 0.419 & 0.404 & 0.359 & 0.324 & 0.301 & 0.279 & 0.274 \\
\hline & 100 & 1 & 0.339 & 0.438 & 0.528 & 0.569 & 0.597 & 0.604 & 0.569 & 0.514 \\
\hline & & $\infty$ & 0.363 & 0.469 & 0.566 & 0.611 & 0.642 & 0.648 & 0.611 & 0.551 \\
\hline & 10 & 1 & 0.100 & 0.131 & 0.159 & 0.174 & 0.183 & 0.186 & 0.177 & 0.159 \\
\hline & & $\infty$ & 0.110 & 0.143 & 0.174 & 0.190 & 0.201 & 0.204 & 0.193 & 0.174 \\
\hline \multirow[t]{8}{*}{100} & 10000 & 1 & 0.090 & 0.072 & 0.042 & 0.036 & 0.036 & 0.036 & 0.035 & 0.036 \\
\hline & & $\infty$ & 0.083 & 0.065 & 0.038 & 0.034 & 0.033 & 0.033 & 0.033 & 0.033 \\
\hline & 1000 & 1 & 0.237 & 0.277 & 0.273 & 0.248 & 0.228 & 0.213 & 0.197 & 0.192 \\
\hline & & $\infty$ & 0.231 & 0.267 & 0.257 & 0.228 & 0.206 & 0.191 & 0.178 & 0.175 \\
\hline & 100 & 1 & 0.289 & 0.374 & 0.451 & 0.487 & 0.511 & 0.516 & 0.487 & 0.439 \\
\hline & & $\infty$ & 0.306 & 0.396 & 0.478 & 0.516 & 0.541 & 0.547 & 0.516 & 0.465 \\
\hline & 10 & 1 & 0.098 & 0.127 & 0.155 & 0.169 & 0.178 & 0.181 & 0.172 & 0.155 \\
\hline & & $\infty$ & 0.108 & 0.141 & 0.172 & 0.187 & 0.198 & 0.201 & 0.190 & 0.171 \\
\hline \multirow[t]{8}{*}{10} & 10000 & 1 & 0.011 & 0.009 & 0.005 & $\overline{0.004}$ & $\overline{0.004}$ & 0.004 & 0.004 & $\overline{0.004}$ \\
\hline & & $\infty$ & 0.011 & 0.008 & 0.005 & 0.004 & 0.004 & 0.004 & 0.004 & 0.004 \\
\hline & 1000 & 1 & 0.047 & 0.055 & 0.054 & 0.049 & 0.045 & 0.042 & 0.038 & 0.037 \\
\hline & & $\infty$ & 0.044 & 0.051 & 0.049 & 0.044 & 0.039 & 0.037 & 0.034 & 0.033 \\
\hline & 100 & 1 & 0.106 & 0.138 & 0.166 & 0.180 & 0.188 & 0.190 & 0.180 & 0.162 \\
\hline & & $\infty$ & 0.107 & 0.139 & 0.167 & 0.181 & 0.190 & 0.192 & 0.181 & 0.163 \\
\hline & 10 & 1 & 0.072 & 0.093 & 0.114 & 0.124 & 0.131 & 0.133 & 0.126 & 0.114 \\
\hline & & $\infty$ & 0.083 & 0.108 & 0.132 & 0.144 & 0.152 & 0.154 & 0.146 & 0.131 \\
\hline
\end{tabular}


T. Meshii, et al., Journal of Thermal Stresses, Vol. 27, No. 3, pp. 253 - 267 (2004. 3).

Table 6 Normalized transient stress intensity factor range $\Delta K_{\text {cyl }} / K_{\Delta T}$ at $1^{\text {st }}$ and $\infty$ cycle $\left(r_{\mathrm{m}} / W=5, H=5 / \beta, v=0.3\right)$.

\begin{tabular}{|c|c|c|c|c|c|c|c|c|c|c|}
\hline \multirow[b]{2}{*}{$B$} & \multirow[b]{2}{*}{$\Omega$} & \multirow[b]{2}{*}{ cycle } & \multicolumn{7}{|c|}{$a / W$} & \multirow[b]{2}{*}{0.7} \\
\hline & & & 0.05 & 0.1 & 0.2 & 0.3 & 0.4 & 0.5 & 0.6 & \\
\hline \multirow[t]{8}{*}{10000} & 10000 & & 0.155 & 0.088 & 0.058 & 0.049 & 0.045 & 0.042 & 0.038 & 0.037 \\
\hline & & $\infty$ & 0.145 & 0.082 & 0.055 & 0.046 & 0.043 & 0.040 & 0.036 & 0.035 \\
\hline & 1000 & 1 & 0.330 & 0.314 & 0.211 & 0.152 & 0.135 & 0.126 & 0.114 & 0.109 \\
\hline & & $\infty$ & 0.325 & 0.303 & 0.195 & 0.140 & 0.127 & 0.120 & 0.108 & 0.104 \\
\hline & 100 & 1 & 0.388 & 0.474 & 0.504 & 0.480 & 0.445 & 0.400 & 0.337 & 0.282 \\
\hline & & $\infty$ & 0.391 & 0.475 & 0.499 & 0.469 & 0.430 & 0.382 & 0.320 & 0.268 \\
\hline & 10 & 1 & 0.277 & 0.357 & 0.421 & 0.438 & 0.437 & 0.416 & 0.363 & 0.297 \\
\hline & & $\infty$ & 0.316 & 0.407 & 0.480 & 0.500 & 0.500 & 0.475 & 0.415 & 0.338 \\
\hline \multirow[t]{8}{*}{1000} & 10000 & 1 & 0.134 & 0.076 & 0.050 & 0.042 & 0.039 & 0.036 & 0.032 & 0.032 \\
\hline & & $\infty$ & 0.124 & 0.070 & 0.047 & 0.039 & 0.036 & 0.034 & 0.031 & 0.030 \\
\hline & 1000 & 1 & 0.314 & 0.300 & 0.201 & 0.145 & 0.128 & 0.120 & 0.108 & 0.104 \\
\hline & & $\infty$ & 0.309 & 0.288 & 0.185 & 0.133 & 0.121 & 0.114 & 0.103 & 0.098 \\
\hline & 100 & 1 & 0.382 & 0.467 & 0.497 & 0.473 & 0.438 & 0.394 & 0.332 & 0.277 \\
\hline & & $\infty$ & 0.385 & 0.468 & 0.491 & 0.462 & 0.423 & 0.376 & 0.315 & 0.263 \\
\hline & 10 & 7 & 0.275 & 0.355 & 0.419 & 0.436 & 0.435 & 0.414 & 0.362 & 0.295 \\
\hline & & $\infty$ & 0.314 & 0.405 & 0.478 & 0.497 & 0.497 & 0.472 & 0.412 & 0.336 \\
\hline \multirow[t]{8}{*}{100} & 10000 & 1 & 0.050 & 0.028 & 0.018 & 0.015 & 0.014 & 0.013 & 0.012 & 0.012 \\
\hline & & $\infty$ & 0.045 & 0.025 & 0.017 & 0.014 & 0.013 & 0.012 & 0.011 & 0.011 \\
\hline & 1000 & 1 & 0.205 & 0.195 & 0.131 & 0.094 & 0.083 & 0.077 & 0.070 & 0.067 \\
\hline & & $\infty$ & 0.196 & 0.183 & 0.118 & 0.084 & 0.077 & 0.073 & 0.065 & 0.063 \\
\hline & 100 & 1 & 0.328 & 0.401 & 0.427 & 0.407 & 0.378 & 0.339 & 0.286 & 0.239 \\
\hline & & $\infty$ & 0.328 & 0.398 & 0.419 & 0.393 & 0.360 & 0.320 & 0.268 & 0.224 \\
\hline & 10 & 1 & 0.261 & 0.337 & 0.397 & 0.413 & 0.413 & 0.393 & 0.343 & 0.280 \\
\hline & & $\infty$ & 0.297 & 0.382 & 0.451 & 0.470 & 0.469 & 0.446 & 0.389 & 0.318 \\
\hline \multirow[t]{8}{*}{10} & 10000 & 1 & 0.006 & 0.003 & 0.002 & 0.002 & 0.002 & 0.002 & 0.001 & 0.001 \\
\hline & & $\infty$ & 0.006 & 0.003 & 0.002 & 0.002 & 0.002 & 0.002 & 0.001 & 0.001 \\
\hline & 1000 & 1 & 0.040 & 0.038 & 0.025 & 0.018 & 0.015 & 0.015 & 0.013 & 0.013 \\
\hline & & $\infty$ & 0.038 & 0.035 & 0.022 & 0.016 & 0.015 & 0.014 & 0.013 & 0.012 \\
\hline & 100 & 1 & 0.123 & 0.151 & 0.161 & 0.154 & 0.143 & 0.128 & 0.108 & 0.090 \\
\hline & & $\infty$ & 0.118 & 0.143 & 0.150 & 0.141 & 0.129 & 0.115 & 0.096 & 0.081 \\
\hline & 10 & & 0.164 & 0.212 & 0.250 & 0.260 & 0.260 & 0.247 & 0.216 & 0.176 \\
\hline & & $\infty$ & 0.177 & 0.229 & 0.270 & 0.281 & 0.281 & 0.267 & 0.233 & 0.190 \\
\hline
\end{tabular}

Table 7 Normalized transient stress intensity factor range $\Delta K_{\text {cyl }} / K_{\Delta T}$ at $1^{\text {st }}$ and $\infty$ cycle $\left(r_{\mathrm{m}} / W=1, H=5 / \beta, v=0.3\right)$.

\begin{tabular}{|c|c|c|c|c|c|c|c|c|c|c|}
\hline \multirow[b]{2}{*}{$B$} & \multirow[b]{2}{*}{$\Omega$} & \multirow[b]{2}{*}{ cycle } & \multicolumn{7}{|c|}{$a / W$} & \multirow[b]{2}{*}{0.7} \\
\hline & & & 0.05 & 0.1 & 0.2 & 0.3 & 0.4 & 0.5 & 0.6 & \\
\hline \multirow[t]{8}{*}{10000} & 10000 & & 0.015 & 0.009 & $\overline{0.004}$ & 0.003 & 0.002 & 0.002 & 0.001 & 0.001 \\
\hline & & $\infty$ & 0.015 & 0.008 & 0.004 & 0.003 & 0.002 & 0.002 & 0.001 & 0.001 \\
\hline & 1000 & 1 & 0.042 & 0.026 & 0.013 & 0.008 & 0.005 & 0.004 & 0.003 & 0.002 \\
\hline & & $\infty$ & 0.040 & 0.025 & 0.012 & 0.008 & 0.005 & 0.004 & 0.003 & 0.002 \\
\hline & 100 & 1 & 0.165 & 0.087 & 0.042 & 0.026 & 0.018 & $\overline{0.012}$ & 0.008 & 0.007 \\
\hline & & $\infty$ & 0.156 & 0.079 & 0.040 & 0.024 & 0.017 & 0.012 & 0.008 & 0.007 \\
\hline & 10 & 1 & 0.329 & 0.301 & 0.172 & 0.089 & 0.054 & 0.037 & 0.025 & 0.020 \\
\hline & & $\infty$ & 0.324 & 0.291 & 0.158 & 0.080 & 0.050 & 0.036 & 0.025 & 0.019 \\
\hline \multirow[t]{8}{*}{1000} & 10000 & 1 & 0.013 & 0.007 & $\overline{0.003}$ & 0.002 & 0.002 & 0.001 & 0.001 & 0.001 \\
\hline & & $\infty$ & 0.012 & 0.007 & 0.003 & 0.002 & 0.002 & 0.001 & 0.001 & 0.001 \\
\hline & 1000 & 1 & 0.040 & 0.025 & 0.012 & 0.008 & 0.005 & 0.004 & 0.003 & 0.002 \\
\hline & & $\infty$ & 0.038 & 0.023 & 0.012 & 0.007 & 0.005 & 0.003 & 0.002 & 0.002 \\
\hline & 100 & 1 & 0.163 & 0.085 & 0.041 & 0.025 & 0.017 & 0.012 & 0.008 & 0.007 \\
\hline & & $\infty$ & 0.154 & 0.078 & 0.039 & 0.024 & 0.017 & 0.012 & 0.008 & 0.007 \\
\hline & 10 & 1 & 0.327 & 0.299 & 0.171 & 0.089 & 0.054 & 0.037 & 0.025 & 0.020 \\
\hline & & $\infty$ & 0.323 & 0.289 & 0.158 & 0.079 & 0.050 & 0.036 & 0.025 & 0.019 \\
\hline \multirow[t]{8}{*}{100} & 10000 & 1 & $\overline{0.004}$ & 0.002 & $\overline{0.001}$ & 0.001 & 0.001 & 0.000 & 0.000 & $\overline{0.000}$ \\
\hline & & $\infty$ & 0.004 & 0.002 & 0.001 & 0.001 & 0.001 & 0.000 & 0.000 & 0.000 \\
\hline & 1000 & 1 & 0.026 & 0.016 & 0.008 & 0.005 & 0.003 & 0.002 & 0.002 & 0.001 \\
\hline & & $\infty$ & 0.025 & 0.015 & 0.008 & 0.005 & 0.003 & 0.002 & 0.002 & 0.001 \\
\hline & 100 & 1 & 0.140 & 0.073 & 0.035 & 0.022 & 0.015 & 0.011 & 0.007 & 0.006 \\
\hline & & $\infty$ & 0.131 & 0.066 & 0.034 & 0.021 & 0.014 & 0.010 & 0.007 & 0.006 \\
\hline & 10 & 1 & 0.311 & 0.284 & 0.162 & 0.084 & 0.051 & 0.035 & 0.024 & 0.019 \\
\hline & & $\infty$ & 0.306 & 0.274 & 0.149 & 0.075 & 0.047 & 0.034 & 0.023 & 0.018 \\
\hline \multirow[t]{8}{*}{10} & 10000 & 1 & 0.001 & 0.000 & $\overline{0.000}$ & 0.000 & 0.000 & 0.000 & 0.000 & 0.000 \\
\hline & & $\infty$ & 0.001 & 0.000 & 0.000 & 0.000 & 0.000 & 0.000 & 0.000 & 0.000 \\
\hline & 1000 & 1 & 0.005 & 0.003 & 0.002 & 0.001 & 0.001 & 0.000 & 0.000 & $\overline{0.000}$ \\
\hline & & $\infty$ & 0.005 & 0.003 & 0.001 & 0.001 & 0.001 & 0.000 & 0.000 & 0.000 \\
\hline & 100 & 1 & 0.052 & 0.026 & 0.013 & 0.008 & 0.005 & 0.004 & 0.003 & 0.002 \\
\hline & & $\infty$ & 0.047 & 0.024 & 0.012 & 0.007 & 0.005 & 0.004 & 0.002 & 0.002 \\
\hline & 10 & 1 & 0.198 & 0.181 & 0.103 & 0.053 & 0.032 & 0.022 & 0.015 & 0.012 \\
\hline & & $\infty$ & 0.190 & 0.171 & 0.093 & 0.047 & 0.029 & 0.021 & 0.015 & 0.011 \\
\hline
\end{tabular}

